\title{
LIFE-LONG VITAMIN D DEFICIENCY, COMMENCING IN UTERO, AFFECTS CARDIAC FUNCTION AND INCREASES THE SUSCEPTIBILITY TO MYOCARDIAL ISCHEMIA/REPERFUSION INJURY IN ADULTHOOD
}

\author{
O. Gezmish ${ }^{1}$, H. Parkington ${ }^{2}$, M. Tare ${ }^{2}$, J.M. Black ${ }^{1}$ \\ ${ }^{1}$ Anatomy and Developmental Biology, ${ }^{2}$ Physiology, Monash University, Melbourne, VIC, Australia
}

Background: Vitamin D deficiency is an emerging health problem. Some ethnic/religious groups are exposed to life-long vitamin D deficiency, beginning in utero.

Aims: To investigate the effect of life-long vitamin D deficiency in adult rats on myocardial capillarisation, cardiac function and the susceptibility to ischemia/reperfusion injury.

Methods: Four week old Sprague-Dawley female rats were fed either a vitamin D deplete or vitamin Dreplete (control) diet for 6 weeks prior to pregnancy, during pregnancy and throughout lactation. Offspring remained on their respective diets until adulthood. Hearts of 16 week old vitamin D deficient and control rats ( $n=8$ /group) were mounted on a Langendorff apparatus and cardiac function and the response to ischemia/reperfusion assessed. In separate cohorts myocardial capillarisation was quantified using stereological techniques.

Results: Basal and stimulated heart function was not altered, although coronary flow was significantly reduced $(\mathrm{p}=0.007)$ in vitamin $\mathrm{D}$ deficient rats. Strikingly, infarct area was 2 -fold greater in vitamin $\mathrm{D}$ deficient hearts of both males and females ( $p=0.006 \& p=0.03$, respectively). Myocardial vascularisation was not different between the groups.

Conclusion: Hearts of vitamin D deficient rats were particularly susceptible to ischemia/reperfusion injury. Dysregulation of coronary flow is likely contributing to the increased susceptibility of ischemia/reperfusion injury, but this is not attributed to myocardial vascularisation. 